\title{
Photopolymer-based volume holographic optical elements: design and possible applications
}

\section{G. Bianco}

\section{A. Ferrara}

antonella.ferrara@na.imm.cnr.it

\section{F. Borbone}

\section{A. Roviello}

\section{Striano}

\section{G. Coppola}

National Research Council, Institute for Microelectronics and Microsystems, Via P. Castellino 111, 80131 Napoli, Italy

National Research Council, Institute for Microelectronics and Microsystems, Via P. Castellino 111, 80131 Napoli, Italy

Department of Chemical Sciences, University of Naples Federico II, Via Cinthia, 80126 Napoli, Italy

Department of Chemical Sciences, University of Naples Federico II, Via Cinthia, 80126 Napoli, Italy

ANTARES S.c.a.r.l., Via Appia 1, 82018 San Giorgio del Sannio (BN), Italy

National Research Council, Institute for Microelectronics and Microsystems, Via P. Castellino 111, 80131 Napoli, Italy

In this paper, Volume Holographic Optical Elements (V-HOEs), such as holographic gratings and spherical lenses, are designed and fabricated by using a prototype of photopolymer. The recording process of V-HOEs and their appropriate characterization are described. Moreover, $\mathrm{V}$-HOEs possible applications as solar concentrator are investigated and results are discussed. Finally, a system that allows passive solar tracking is proposed and preliminary results are reported.

[DOI: http://dx.doi.org/10.2971/jeos.2015.15057]

Keywords: Holographic lens, holographic grating, volume phase holographic optical elements, solar concentrator

\section{INTRODUCTION}

In the last years, Volume Holographic Optical Elements (VHOEs) have attracted research efforts, due to their promising use in many applications. Indeed, V-HOEs can improve the benefits provided by classical surface-relief grating technology and optics, allowing theoretical diffraction efficiency that could even reach $100 \%$ at the wavelength for which V-HOE was designed [1]. V-HOEs are based on diffraction of the light in several directions through the bulk refractive index modulation obtained by the recording process $[2,3]$.

It is important to underline that volume holography is actually considered desirable for various applications. For example, complex grating structures can be produced to minimize optical elements in some spectrograph configurations, simplifying spectrograph design and enhancing spectrograph efficiency [1]. Additionally, transmitting Volume Holographic Grating (VHG) recorded within a photopolymer appositely functionalized to detect a specific stimulus or analyte can be used as sensor holograms [4]. Therefore, the realization of high efficient V-HOEs is a topic of growing interest.

Moreover, due to their high efficiency, low cost, and to their capability to redirect, concentrate or block the incident light, holographic planar optics have recently been developed to integrate the same functionalities of conventional solar concentrators [5]; additionally, V-HOEs are thin, flat and lightweight, all very important features for solar concentrators. Another important point is related to the possibility to multiplex several holographic elements into the same panel, allowing collecting solar rays with different incidence angles. This means that a V-HOE, such as a holographic lens, can redirect the light coming from a moving source, such as the sun, thus reducing mechanical movements and implementing a passive solar tracking. As a consequence, with the perspective of new generation solar panel development, the use of V-HOEs could lead to a very competitive system [6].

HOEs were suggested to be used in solar applications for the first time in 1982 [7]. Afterwards, a variety of designs have been suggested over the years [8,9]. For example, was demonstrated that a volume holographic lens allows a single-axis tracking over 55 degree angular variation [10,11]. Designs of multiplexed holographic lenses have been also proposed, but the diffraction efficiency typically dropped passing from the recording of a single element to stacked elements [6].

In this paper, we describe the recording process to obtain VHOEs, afterwards their characterization is reported. As sensitive substrate, we used a prototype material and for the recording of $\mathrm{V}$-HOEs we used a typical holographic interferometry configuration. In particular, two different types of optical elements were carried out and characterized: Volume Holographic Grating (VHG) and Volume Holographic Lens (VHL). Their use, are discussed with particular care to the 
solar applications, too. Finally, a multiplexed volume holographic lenses configuration is proposed to implement a passive solar tracking.

\section{RESULTS AND DISCUSSION}

V-HOEs have been recorded on a prototype of photopolymer deposited (thickness $\approx 30 \mu \mathrm{m}$ ) by the Doctor Blade method on a rigid support [12]. The final refractive index modulation of the photopolymer, induced by a green light and fixed by a post writing photo-bleaching step, was of about 0.02 [12]. The optical set-up used for the recording of V-HOEs was a typical interferometer and as recording source, a laser emitting at $532 \mathrm{~nm}$ (green source) was used. The interference pattern between the reference and object beams has been recorded on the photopolymer and the exposure time was 3.5 minutes. The diameter of the recorded hologram was about $4 \mathrm{~cm}$. As optical elements, we have recorded both VHG and VHL.

Regarding VHG, Figure 1 shows the angular selectivity curves obtained both experimentally and by the simulated theory values according to the Kogelnik's coupled wave theory [2]. The FWHM of the curve reported in Figure 1 gives a grating angular selectivity of about $2^{\circ}$. The diffraction efficiency $\eta$ was of about $94 \%$ and it has been calculated as:

$$
\eta=P_{1} /\left(P_{\text {inc }}-P_{\text {refl }}\right)
$$

where $P_{1}$ is the power of the $1^{\text {st }}$ diffraction order, $P_{\text {inc }}$ and $P_{r e f l}$ are the incident and the reflected powers, respectively. An Atomic Force Microscopy (AFM) characterization on the VHG surface shows a small surface modulation of about $5 \mathrm{~nm}$, maybe due to shrinkage of the material. Thus a formation of a surface grating was observed (see Figure 2). However, a confirmation that in our VHG the volume effect is predominant is given by the $\mathrm{Q}$ parameter, defined as:

$$
Q=\frac{2 \pi \lambda t}{\left(n \Lambda^{2}\right)}
$$

where $\lambda$ is the recording wavelength, $t$ is the photosensitive layer thickness, $n$ is the refractive index of the material, and $\Lambda$ is the fringe spacing. A holographic grating is considered to be a surface hologram when $Q \leq 1$, a volume hologram when $Q \geq 10$ [13]. In our case, we obtained a $Q \approx 17$, therefore our hologram is a VHG and the surface grating observed by AFM can be neglected.

In the case of a VHL, a single lens was recorded with an in-line configuration by using a concave mirror with a focal length of $5 \mathrm{~cm}$ as object. The refractive index modulation has been generated by interference between a plane (reference) and a spherical (object) wavefronts. The obtained VHL was characterized and its efficiency has been evaluated as the ratio between the power focused by the holographic lens and the power focused by a commercial Fresnel lens with the same focusing features. About 40 lenses have been recorded and for each one the efficiency has been measured. The extrapolated value for the efficiency was $41.49 \% \pm 1.35 \%$. Furthermore, each lens has been characterized at different angles of incidence, and an angular selectivity of about $\pm 8^{\circ}$ was obtained.

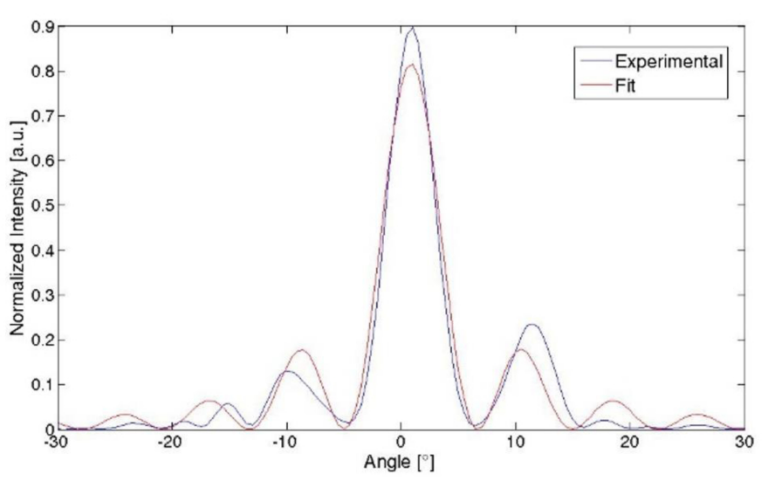

FIG. 1 Angular selectivity curve.

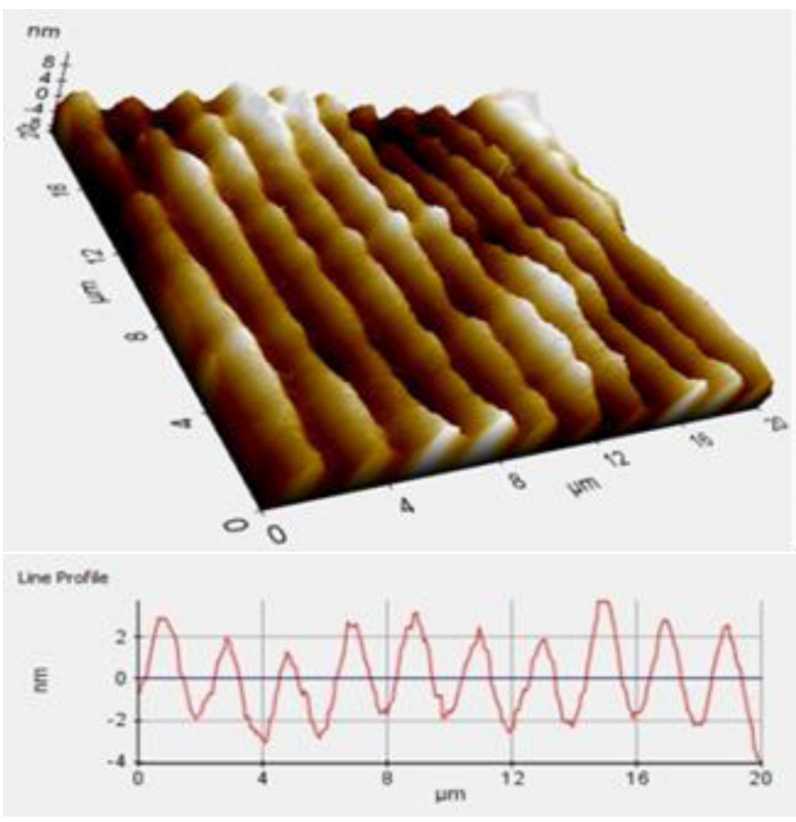

FIC. 2 a) AFM characterization of the surface of a VHG; b) line profile.

With the aim to implement a passive solar tracking, multiplexed holographic lenses have been designed and recorded. This structure allows directing the light in a fixed direction independently of the direction of the incoming light without any mechanical movement.

In particular, a set of three lenses $\left(0^{\circ}, \pm 30^{\circ}\right)$ has been recorded on the same glass to focus the light on the same PV cell positioned close to the focus of the lenses system. The recording procedure was implemented for three angles: perpendicular illumination $\left(0^{\circ}\right),+30^{\circ}$ illumination and $-30^{\circ}$ illumination. Holographic lenses at $\pm 30^{\circ}$ were recorded by tilting of $\pm 30^{\circ}$ the photosensitive substrate. In Figure 3, the passive solar tracking collection is conceptually illustrated.

The three multiplexed holographic lenses system has been characterized in terms of degree of concentration, expressed in number of "suns". This parameter is evaluated as the ratio between the intensity of the light harvested by the PV cell with and without the solar concentrator. Preliminary results show a degree of concentration for the proposed system on average of 5.85 suns over the angular range of $\pm 30^{\circ}$. For comparison, the same characterization was performed on a single holographic lens, and a degree of concentration of about 5.5suns 


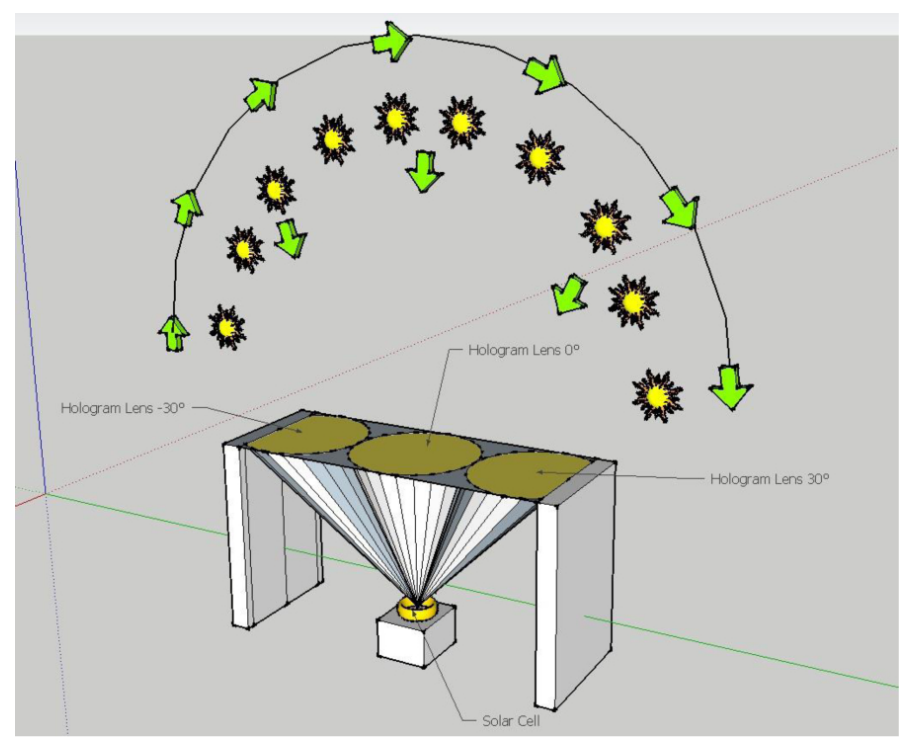

FIC. 3 Schematic representation of passive solar tracking implemented by three multiplexed VHL.

was obtained for an angular incidence inside the lens angular selectivity range $\left( \pm 8^{\circ}\right)$, therefore the multiplexing systems enhances the angular acceptance.

\section{CONCLUSIONS}

In this work, high efficiency transmitting VHG and VHL are recorded by using a new photopolymer sensitive to green light. The experimental results prove a diffraction efficiency for VHG of about $94 \%$, whereas the grating angular selectivity is $2^{\circ}$. Evaluation of the Q-parameter confirms the volume effect. Regarding VHL, the obtained mean efficiency, calculated as the ratio between the power focused by the holographic lens and the power focused by a commercial Fresnel lens, is of $41.49 \%$ and the angular selectivity is of $\pm 8^{\circ}$. Furthermore, the versatility of holographic technology permits to cluster multiple holographic elements into the same panel, collecting solar rays with different incidence angles, allowing, in theory, to develop the holographic passive solar tracker. A multiplexing system of three holographic lenses was recorded in order to avoid mechanical movements and a degree of concentration of 5.85suns was obtained for angular incidence in the range of $\pm 30^{\circ}$. Further study in order to increase both the degree of concentration and the angular selectivity are still in progress. However, our results show the possibility to realize solar concentrators for PV cells by using multiplexed VHL with a good efficiency and passive solar tracking.

\section{ACKNOWLEDGEMENTS}

The research activity is supported by Italian Ministry of Education, University and Research (MIUR) through the PANDION project, in the framework of the National Operational Program - PON.

\section{References}

[1] S. C. Barden, J. A. Arns, and W. S. Colburn, "Volume-phase holographic gratings and their potential for astronomical applications," Proc. SPIE 3355, 866 (1998).

[2] H. Koegelnik, "Coupled wave theory for thick hologram gratings," Bell Syst. Tech. J. 48, 2909-2947 (1969).

[3] J. W. Goodman, Introduction to Fourier Optics, 2nd ed., Mc GrawHill, New York, USA, 1996.

[4] G. Bianco, M. A. Ferrara, F. Borbone, F. Zuppardi, A. Roviello, V. Striano, and G. Coppola, "Volume holographic gratings as optical sensor for heavy metal in bathing waters," Proc. SPIE 9506, 95062B (2015).

[5] W. H. Bloss, M. Griesinger, and E. R. Reinhardt, "Dispersive concentrating systems based on transmission phase holograms for solar applications," Appl. Optics 21, 3739-3742 (1982).

[6] I. Naydenova, H. Akbari, C. Dalton, M. Yahya, M. Ilyas, C. P. T. Wei, V. Toal, et al., "Photopolymer Holographic Optical Elements for Application in Solar Energy Concentrators" in Holography - Basic Principles and Contemporary Applications, E. Mihaylova, ed., 129145 (InTech, Rijeka, 2013).

[7] J. E. Ludman, "Holographic solar concentrator," Appl. Optics 21, 30573058 (1982).

[8] Y. W. Zhang, C. S. Ih, H. F. Yan, and M. J. Chang, "Photovoltaic concentrator using a holographic optical element," Appl. Optics 27, 3556-3560 (1988).

[9] J. A. Quintana, P. G. Boj, J. Crespo, M. Pardo, and M. A. Satorre, "Line-focusing holographic mirrors for solar ultraviolet energy concentration," Appl. Optics 36, 3689-3693 (1997).

[10] D. Chemisana, M.V. Collados, M. Quintanilla, and J. Atencia, "Holographic lenses for building integrated concentrating photovoltaics," Appl. Energ. 110, 227-235 (2013).

[11] X. Ren, S. Liu, X. Zhang, X. Chen, "Fabrication of holographic Fresnel lens used as solar concentrator," in Proceedings to Holography and Diffractive Optics III, 1-7 (SPIE, Beijing, 2007).

[12] M. A. Ferrara, F. Borbone, V. Striano, and G. Coppola, "Characterization of photopolymers as optical recording materials by means of digital holography microscopy," Proc. SPIE 8792, $87920 Z$ (2013).

[13] B. C. Kress, P. Meyureis, Applied Digital Optics: from micro-optics to nanophotonics (John Wiley at Sons, Haboken, 2009). 